\section{V-ATPase as a mediator of treatment resistance in Glioblastoma Multiforme: An in vitro study to investigate new therapeutic strategies}

\author{
Eleonora Messuti, ${ }^{1}$ Martina Giambra, ${ }^{1}$ \\ Serena Redaelli, ${ }^{1}$ Andrea Di Cristofori, ${ }^{2}$ \\ Carlo Giussani, ${ }^{2}$ Angela Bentivegna ${ }^{1}$ \\ 1 University of Milano-Bicocca, School of \\ Medicine and Surgery, Monza; 2 San \\ Gerardo Hospital, ASST Monza, \\ Neurosurgery Unit, Monza, Italy
}

\begin{abstract}
Recent evidences suggest the involvement of the Vacuolar H+ ATPase (VATPase) in the development and/or progression of Glioblastoma Multiforme (GBM). This proton pump could be a valid therapeutic target but more in-depth studies are necessary. The aim of this study is to better define the in vitro effects on Glioma Stem Cell (GSC) primary cultures viability of single and combined treatment with Bafilomycin-A1 (Baf-A1), a V-ATPase inhibitor, and Temozolomide (TMZ), the chemotherapeutic agent currently used to treat GBM patients. We found out that GSC were resistant to $\mathrm{TMZ}$ and more sensitive to treatments with Baf-A1 and that the two drugs exerted a synergistic effect when administered together.
\end{abstract}

\section{Introduction}

Glioblastoma Multiforme (GBM) is a grade IV astrocytoma and is the most common and malignant primary tumor of the central nervous system. ${ }^{1}$ GBM patients have a poor median overall survival of 1416 months after gross total surgical resection and adjuvant chemo-radiation therapy. ${ }^{2}$ The failure of current therapies is mainly due to the striking inter- and intratumoral heterogeneity and to the presence within the tumor mass of cells with stem-like properties, called Glioma Stem Cells (GSCs). GSCs are crucial for tumor growth and response to therapy and we need a deeper understanding of how selectively target and ablate these cell population. ${ }^{3}$ GSCs from patient-derived biopsy samples are the most straightforward, reliable and reproducible in vitro model currently available to study GBM, as they derive from patients and resemble the tumor-of-origin the most. ${ }^{4}$ In
Glioma dysregulated ion balance is emerging as a key factor that could contribute to rapid and aggressive tumor formation and relapse. Specifically, alkalinization of the cytosol and acidification of the extracellular space allows GSCs to evade apoptosis, and increases multidrug resistance, cell proliferation, migration and invasion. ${ }^{5}$ The Vacuolar $\mathrm{H}^{+}$-ATPase (V-ATPase) is an ATPdependent proton pump of the endomembrane system of all eukaryotic organisms. This multisubunit nano-motor acidifies subcellular compartments and, in certain specialized tissues, the extracellular space and is essential for vital cellular processes. In addition, recent evidence indicates that VATPase acts directly in the endolysosomal compartment to modulate dysregulated pathways in cancer, such as Notch, Wnt or mTOR signaling. 6 This evidence, together with the fact that a variety of human tumors, including GBM, displays overexpression of V-ATPase subunits, suggests that V-ATPase might play a crucial, albeit largely unexplored, role in supporting tumorigenesis. So far, overexpression of V-ATPase in human tumors has been associated with invasive and chemoresistant phenotypes and to increased proliferative activity making $\mathrm{V}$ ATPase a potential drug target. ${ }^{7}$

\section{Materials and Methods}

GSCs isolation from tumor biopsies: GBM tumor biopsies were obtained
Correspondence: Eleonora Messuti, University of Milano-Bicocca, School of Medicine and Surgery, Monza, Italy.

E-mail: e.messuti@campus.unimib.it

Key words: GBM; GSC; V-ATPase; Baf-A1.

Acknowledgments: The authors thank Clarissa Cavandoli for her support and all the patients.

Disclosures: The authors have nothing to disclose.

Conference presentation: This paper was presented at the Third Centro 3R Annual Meeting - L'era delle 3R: modelli in silico, in vitro e in vivo per promuovere la ricerca traslazionale 30 September - 1 October 2021, Evento online organizzato dal Politecnico di Torino.

Received for publication: 9 July 2021.

Accepted for publication: 7 September 2021.

This work is licensed under a Creative Commons Attribution NonCommercial 4.0 License (CC BY-NC 4.0).

(C) Copyright: the Author(s), 2021

Licensee PAGEPress, Italy

Biomedical Science and Engineering 2021; 4(s1):182 doi:10.4081/bse.2021.182

from patients undergoing surgery. We optimized a method for the isolation of stem cells from tissue specimens. Briefly: a mechanical and enzymatic

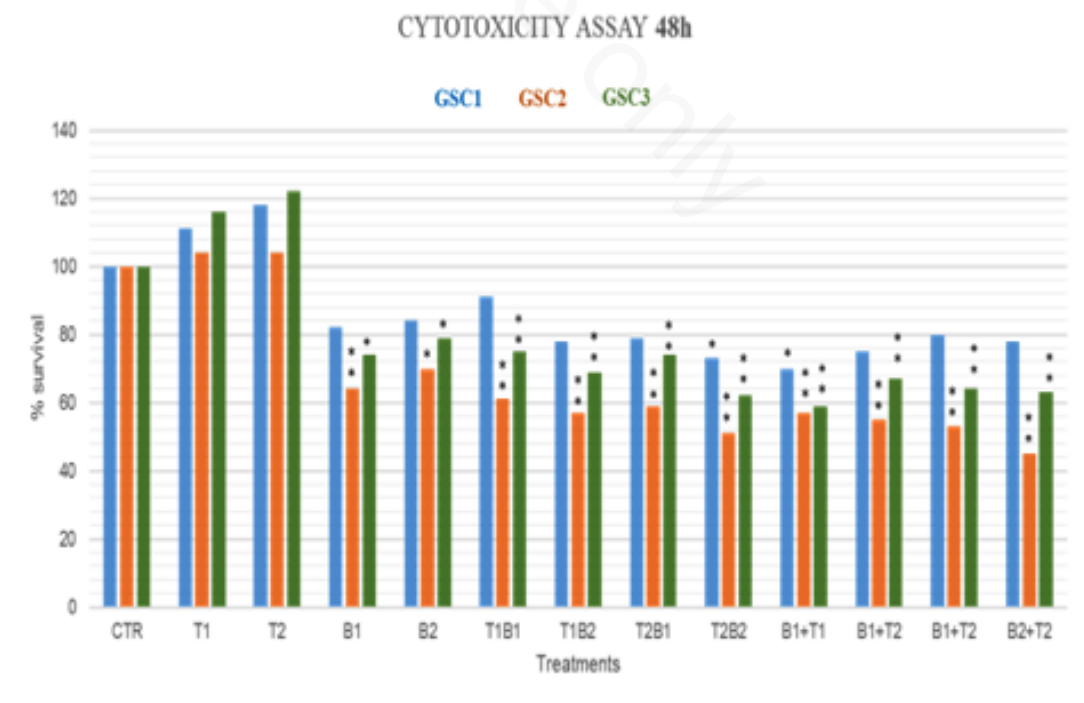

Figure 1. Data are expressed as percentage of viable cells relative to the control (untreated cells). T-Student test were calculated on raw data ${ }^{*}, \mathbf{p}<0,05 ;{ }^{* *}, \mathbf{p}<0,01$. From left to right: CTRL=control; T1=TMZ $100 \mu \mathrm{M}$; T2=TMZ $200 \mu \mathrm{M}, \mathrm{B} 1=\mathrm{Baf}-\mathrm{A} 120 \mathrm{nM}, \mathrm{B} 2=\mathrm{Baf}-$ A1 $40 \mathrm{nM}, \mathrm{TB}=\mathrm{TMZ}$ and Baf-A given simultaneously, B+T=Baf-A1 for 24 hours and then TMZ for 24 hours. 
digestion step was followed by washing, centrifugation and cell strainer passage step and finally a red blood cell lysis. GSCs were cultured in a selective medium for neural stem cells, whose key features are the lack of serum and the presence of growth factors (EGF and FGF).

- GSCs characterization: 1. evaluation of their self-renewal efficiency with clonal assay 2. evaluation of their multipotency capability by performing IF with neural differentiation markers (G-FAP, BIII-Tubulin, MBP).

- In vitro drug response test: GSCs were treated with TMZ and Baf-A1 either alone or combined, simultaneously or at deferred times. The number of viable cells and the percentage of survival after treatments was evaluated using
Cell Counting Kit-8 (CCK-8, SigmaAldrich).

- Synergy Finder online program was used to evaluate the synergistic activity of TMZ and Baf-A1.

\section{Results}

Our results show that inhibition of $\mathrm{V}$ ATPase by Baf-A1 is highly effective on cell viability of GSC primary cultures and that a statistically significant and higher cell death is observed under combined treatment of Baf-A1 and TMZ (Figure 1). Furthermore, TMZ and Baf-A1 proved to have a synergistic effect together with a higher synergy score under combined treatment at deferred times (Figure 2). a. Bliss synergy scoue: 9.969

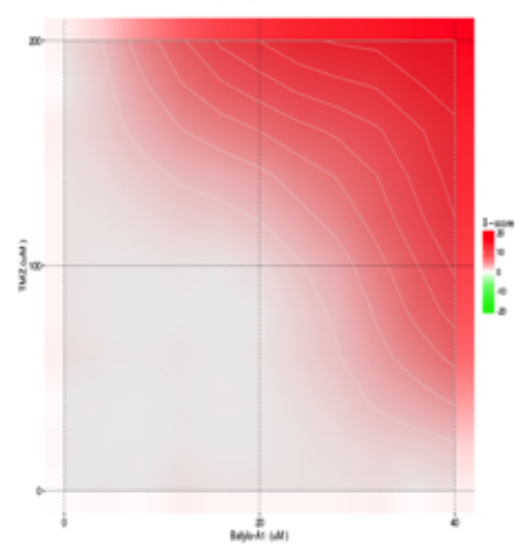

b. Bliss synergy scowe: 17559

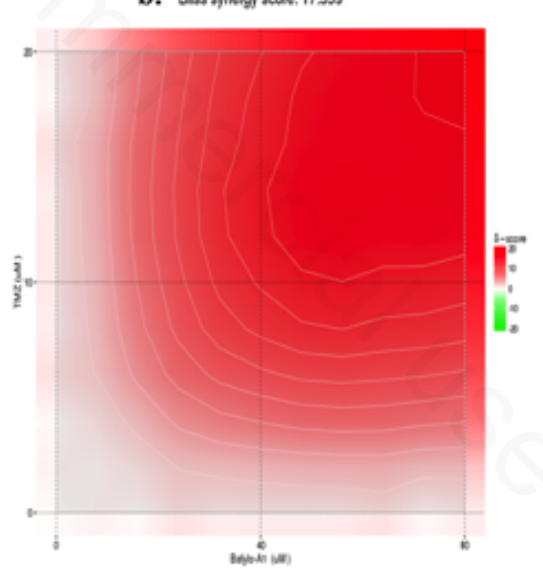

Figure 2. Images show synergy scores as the average excess response due to Baf-A1 (Xaxis) and TMZ (Y-axis) interaction compared to the Bliss independence reference model. Red areas represent synergy (synergy score $>+10$ ). Synergy scores of (a) simultaneous combined treatments (9.969) and (b) at deferred times (17.559).

\section{Discussion and Conclusions}

In conclusion, this work allowed the development of a good method to isolate the stem cell component from biopsy samples and to grow, expand and characterize GSC primary cultures. Data obtained from drug response tests represent a good starting point for understanding the antitumor activity of V-ATPase inhibition in sensitizing GSCs to TMZ therapy. So, in the future, this study could lead to the development of a new target therapy for a better control of this lethal tumor.

\section{References}

1. Louis DN, Perry A, Reifenberger G, et al. The 2016 World Health Organization Classification of Tumors of the Central Nervous System: a summary. Acta Neuropathol 2016;131:803-20.

2. Martínez-Garcia M, Álvarez-Linera J, Carrato C, et al. SEOM clinical guidelines for diagnosis and treatment of glioblastoma (2017). Clin Transl Oncol 2018;20:22-28.

3. Chen J, Li Y, Yu TS, et al. A restricted cell population propagates glioblastoma growth after chemotherapy. Nature 2012;488:522-6.

4. da Hora CC, Schweiger MW, Wurdinger T, Tannous BA. Patient-derived glioma models: from patients to dish to animals. Cells 2019;8:1177.

5. Stransky L, Cotter K, Forgac M. The Function of V-ATPases in Cancer. Physiol Rev 2016;96:1071-91.

6. Vasanthakumar T, Rubinstein JL. Structure and Roles of V-type ATPases. Trends Biochem Sci 2020;45:295-307.

7. Whitton B, Okamoto H, Packham G, Crabb SJ. Vacuolar ATPase as a potential therapeutic target and mediator of treatment resistance in cancer. Cancer Med 2018;7:3800-11. 